Research Article

\title{
Study on Rock Mass Stability Criterion Based on Catastrophe Theory
}

\author{
Zhang Jixun, ${ }^{1}$ Shu Jiaqing, ${ }^{2}$ Zhang Haibo, ${ }^{3}$ Ren Xuhua, ${ }^{1}$ and Qi Jiang ${ }^{1}$ \\ ${ }^{1}$ College of Water Conservancy and Hydropower, Hohai University, No. 1, Xikang Road, Nanjing 210098, China \\ ${ }^{2}$ Jiangsu Electric Power Design Institute, Nanjing, China \\ ${ }^{3}$ Qingdao Municipal Engineering Administration Department, Qingdao, China
}

Correspondence should be addressed to Zhang Jixun; hhu_zhangjx@163.com

Received 19 August 2014; Revised 30 September 2014; Accepted 1 October 2014

Academic Editor: Song Cen

Copyright (C) 2015 Zhang Jixun et al. This is an open access article distributed under the Creative Commons Attribution License, which permits unrestricted use, distribution, and reproduction in any medium, provided the original work is properly cited.

\begin{abstract}
In rock mass engineering, the criterion of rock mass stability has complex nonlinear characteristics, so the process of instability for local rock mass system cannot be quantified by the traditional ways of displacement criterion and the criterion of development of plastic zones, which are strongly empirical. Based on the research about the criterion of rock mass stability, criterion of improved strain energy catastrophe is put forward by virtue of catastrophe theory in this paper. After regularizing potential function, the stability of the system can be determined by catastrophe characteristic values. Take a certain slope for example; the results show that the criterion can quantitatively reflect the behavioral process of instability for rock mass system, which is consistent with the engineering practice and possesses a certain engineering reference value.
\end{abstract}

\section{Introduction}

The stability of surrounding rock is an important criterion to determine the reasonable design and operation safety of the underground rock engineering, for example, the tunnels. Due to the extremely complex rock behavior and occurrence condition and numerous factors, which may influence the stability of rock mass and have features of diversity, variability, and uncertainty, criterion of rock mass stability has not yet reached a mature stage; that is, a series of basic problems have not formed a definite system, from the definition of stability and the criterion of quantization to the theory, the criterion, and the method of analysis [1].

Instability of surrounding rock which is a fairly complex process, often accompanied by heterogeneity, discontinuity, and large displacement of deformation, is a highly nonlinear scientific problem and nonlinearity is the essential characteristics of mechanical behavior of surrounding rock. Therefore, to forecast and control its mechanical behavior, modern nonlinear science is applied. Since the 1970s, developed nonlinear theory, such as fractal, bifurcation, mutation, chaos, and dissipation theory, has become a powerful tool to solve the problem of complicated nonlinear system and to provide theory and method to the nonlinear problem of rock mass. Among them, the application of catastrophe theory established by Thom is used more frequently. Since founded, catastrophe theory has been widely used. It can not only be applied to the "hard science" such as physics, but can also be in the "soft science" like sociology. There exists "a gray area" between soft disciplines and hard disciplines; most of rock mechanics and geoscience belong to this area. Catastrophe theory belongs to qualitative application in rock mechanics. Shan [2] has forecast the possibility of roadway rock burst by the application of catastrophe theory. Yan and $\mathrm{Xu}$ [3] have analyzed the instability of deep-buried hard rock tunnels based on catastrophe theory. Wang and Miao [4] have studied the instability mechanism of pillar by adopting cusp catastrophe model. Xu et al. $[5,6]$ have revealed the process and failure mechanism of rock mass stability and established entropy catastrophe criterion of rock mass instability failure by applying nonlinear scientific theory such as entropy and catastrophe to surrounding rock stability study. Pan et al. [7] have made an exhaustive study on the catastrophe theory applied in the dynamic instability for rock mass system, put 
forward several new ideas and new methods, and published their research works.

\section{Strain Energy Mutation Criterion of Rock MASS Stability}

It has been proven by Thom that when the number of state variable is no more than two and control variable is not greater than four, there will be seven different basic catastrophe types, including fold catastrophe, cusp catastrophe, swallowtail catastrophe, butterfly catastrophe, hyperbolic umbilical catastrophe, elliptic umbilical catastrophe, and parabolic umbilical catastrophe. The first two are simpler and more widely used. In this paper, cusp catastrophe is improved to be applied.

There are two control variables and one state variable in the cusp catastrophe model. Its potential function is

$$
V(x)=x^{4}+u x^{2}+v x .
$$

Similarly with fold catastrophe, equilibrium surface $M$ and sets of crossing points in cusp catastrophe model can be gotten (Figure 1), respectively:

$$
\begin{gathered}
4 x^{3}+2 u x+v=0, \\
8 u^{3}+27 v^{2}=0 .
\end{gathered}
$$

Equilibrium surface equation is a cubic equation and whether it has a real root or 3 real roots depends on the discriminant $\Delta=8 u^{3}+27 v^{2}$. If $\Delta>0$, there will be only one real root and it will be in stable equilibrium. If $\Delta=0$, there will be two different real root $(u, v \neq 0)$ or three same real roots $(u=v=0)$. If $\Delta<0$, there will be three different real roots: two of them which can make $V$ achieve minimum value are stable equilibrium point and the one which makes $V$ achieve maximum value is unstable.

A cubic polynomial is used to fit equilibrium surfaces of system. Based on it, transpose the polynomial to the integral to obtain the potential function of the system. Then regularize it and determine the stability of the system by catastrophe characteristic values.

Take strain energy catastrophe model for example; according to actual excavation step, increment series of strain energy of the system are obtained by numerical calculation, $\{E\}=\{E(1), E(2), \ldots, E(m)\}$. Fit the increment series into the form of polynomial through the least square method. However, the differences lie in the following two aspects.

(1) The variables and the independent variables of the fitting polynomial change. The increment of strain energy is an independent variable and the excavation parameter is a variable.

(2) Adopt a cubic polynomial to fit.

The fitting polynomial is

$$
m=b_{3} E^{3}+b_{2} E^{2}+b_{1} E+b_{0}
$$

$b_{i}$ is the fitting factor of the polynomial. $m$ is the excavation step.

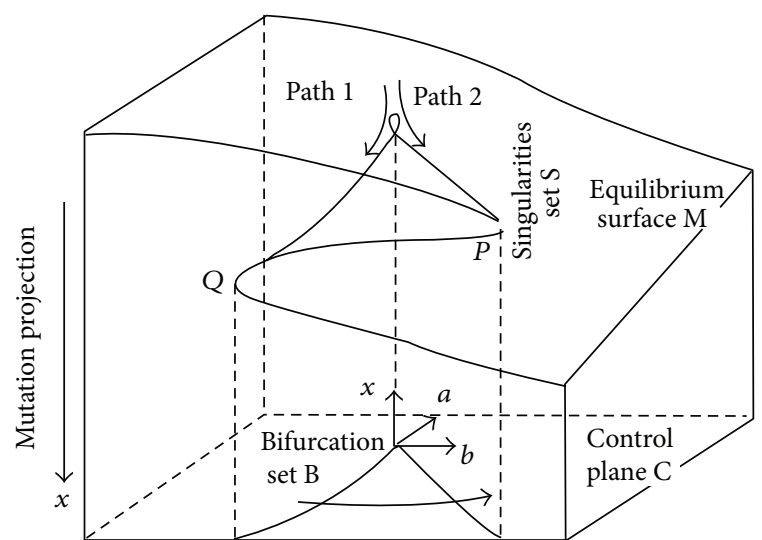

FIGURE 1: Equilibrium surface and sets of crossing points in cusp catastrophe.

Consider the fitting formula as the manifolds of potential function of system (equilibrium surface).

Transposition and integration are as follows:

$$
\frac{b_{3}}{4} E^{4}+\frac{b_{2}}{3} E^{3}+\frac{b_{1}}{2} E^{2}+\left(b_{0}-m\right) E+\Lambda=0 ;
$$

$\Lambda$ is a constant term.

Choose the following as a potential function of the system:

$$
V(E)=\frac{b_{3}}{4} E^{4}+\frac{b_{2}}{3} E^{3}+\frac{b_{1}}{2} E^{2}+\left(b_{0}-m\right) E .
$$

When researching the stability of the system at excavation step $m, V^{\prime}(E) \approx 0$, the system is near the state of equilibrium; the difference between $V^{\prime}(E)$ and 0 is just caused by the fitting polynomial which is not always totally accurate at the fitting point. Thus, it meets the requirements of the neighborhood status near the critical point when studying in catastrophe theory. Meanwhile, parameter $m$ is a known value, so the standard type of cusp catastrophe model can be gotten by means of regularization of the above formula and stability of the system can be determined by catastrophe characteristic values. The system state variable-increment of strain energy can produce a sudden jump when system mutates, which has clear and reasonable physical meaning.

It must be pointed out that the quartic polynomial fitting about the excavation parameter $m$ and the increment of strain energy cannot be established directly. Otherwise, in the view of mathematics, the potential function of the system is the excavation parameter. On this occasion, increment of strain energy $x$ will produce a sudden jump when system instability occurs and will lead to a sudden jump of the potential function of the system; that is to say, the excavation parameter produces a sudden jump which does not conform to the fact that the influence of excavation parameters on system stability should be reflected in control variables.

\section{Examples}

3.1. The Stability Analysis of Slope Based on the Existing Displacement Catastrophe Criterion. A slope is composed of 
TABLE 1: Control variables $u, v$ and catastrophe characteristic value $\Delta$ of cusp catastrophe model under different reduction factors.

\begin{tabular}{lcccccc}
\hline$k$ & 1.2 & 1.25 & 1.3 & 1.31 & 1.32 & -0.015538 \\
\hline$u$ & 0.07375 & -0.00897 & -0.00747 & -0.00949 & -0.01948 \\
$v$ & 0.138 & 0.003068 & 0.004682 & 0.002863 & 0.0013598 & 0.00069 \\
$\Delta$ & 0.517397 & 0.000248 & 0.000588 & 0.000214 & $1.992 E-05$ & $-4.6 E-05$ \\
\hline State & No mutation & No mutation & No mutation & No mutation & No mutation & Mutation \\
\hline
\end{tabular}

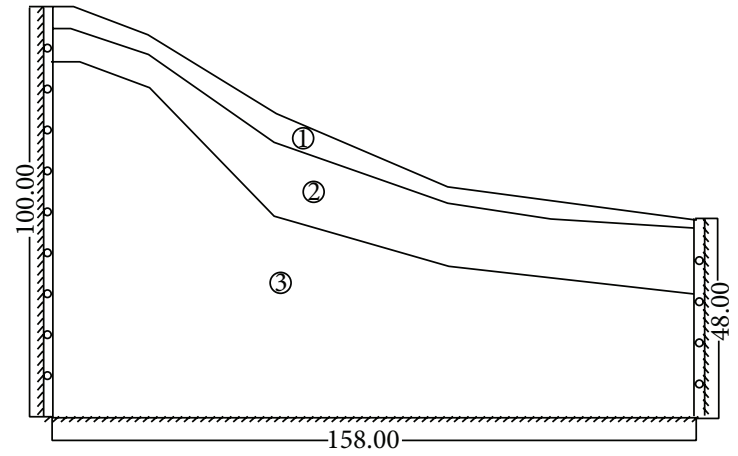

(1) Drift bedding clay soil

(2) Granulite residual clay soil

(3) Weathered granulite

Figure 2: Geometric model and boundary conditions of a slope.

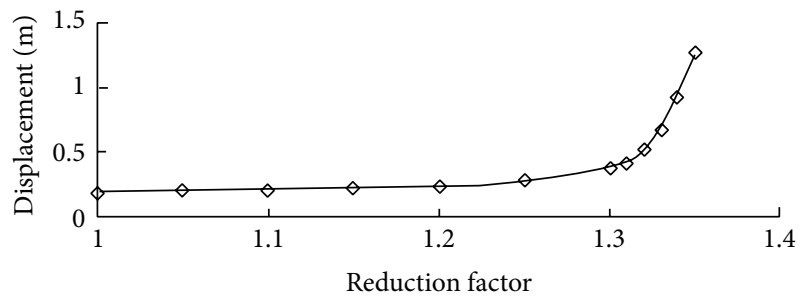

FIGURE 3: Relation curve graph of maximum displacement and reduction factor.

three rock-soil layers. Its geometric size and boundary conditions are shown in Figure 2. Specific parameter characteristics can be found in [8].

According to strength reduction method, the direct relationship between the maximum displacement of the slope and the strength reduction factor is gotten by continuously reducing material parameters and conducting conventional elastic-plastic finite element calculation, as is shown in Figure 3. (The author of [8] has completed the finite element calculation and provided the maximum displacement of the slope under different reduction factors. Discussion and analysis are made on the basis of [8]. Thanks should be given to the author of [8]!)

A total displacement sequence can be gotten:

$$
\{U\}=\{0.1798,0.1928,0.2066,0.2212,0.2369,0.2812,
$$

$$
0.3678,0.418,0.5142,0.6732,0.9171,1.264\} \text {. }
$$

Displacement sequence under different reduction factors is the subset of the total displacement sequence. Analyze displacement sequence under the reduction factor at all levels with least square method to fit it into quartic polynomial (at least from level 5) and then regularize the polynomial to get standard form of catastrophe model.

The fitting relationship between the maximum displacement and reduction factor of slope under reduction factor at levels 5 to 10 and the fitting formula of quartic polynomial with the square value of the correlation coefficient are given in Figure 4. As is shown in the above, applying quartic polynomial fitting can meet the demand of high accuracy. After turning the quartic polynomial fitting into a standard form of cusp catastrophic model to get control variable values under the reduction factor at different levels, corresponding catastrophe characteristic value can be gotten, as is shown in Table 1.

It can be found in the table that the situation of the system will mutate when the reduction factor is 1.33 . Therefore, it can be concluded that the safety coefficient of the slope stability is 1.32 and, according to the cusp catastrophe model, the system characteristic values of mutation are $\Delta=1.992 E-5$ which is very small and close to the edge of the instability.

Spencer method in the traditional limit equilibrium slice method is also used to verify the calculation results in [8]. According to Spencer method, the slope safety factor is 1.29, which is close to 1.32 of the catastrophic model established in this paper, and the error is only $2.3 \%$. It means the result of cusp catastrophe model is of some reliability.

In the model above, based on potential function of the maximum displacement of the slope, the cusp catastrophic model is established. From the fitting formula and the regularized equation, it is known that the state variable $x$ of the model is the strength reduction factor of the slope material. Based on catastrophe theory, there will be a jump when the slope is instable; correspondingly there will be a jump of the strength reduction factor in this model. However, the strength reduction factor is a constant man-made value. Therefore, the conventional displacement catastrophe criterion cannot explain practical meaning. In order to solve this problem, the catastrophic model is proposed and analyzed in this paper.

\subsection{Analysis of Slope Stability Based on the Displacement} Catastrophe Criterion Established in This Paper. When adopting the displacement criterion to determine the stability of slope rock mass, it would certainly bring out displacement catastrophe at the time of slope failure, which is also consistent with the practical engineering experience. Therefore, take the maximum displacement of the slope as state variable 

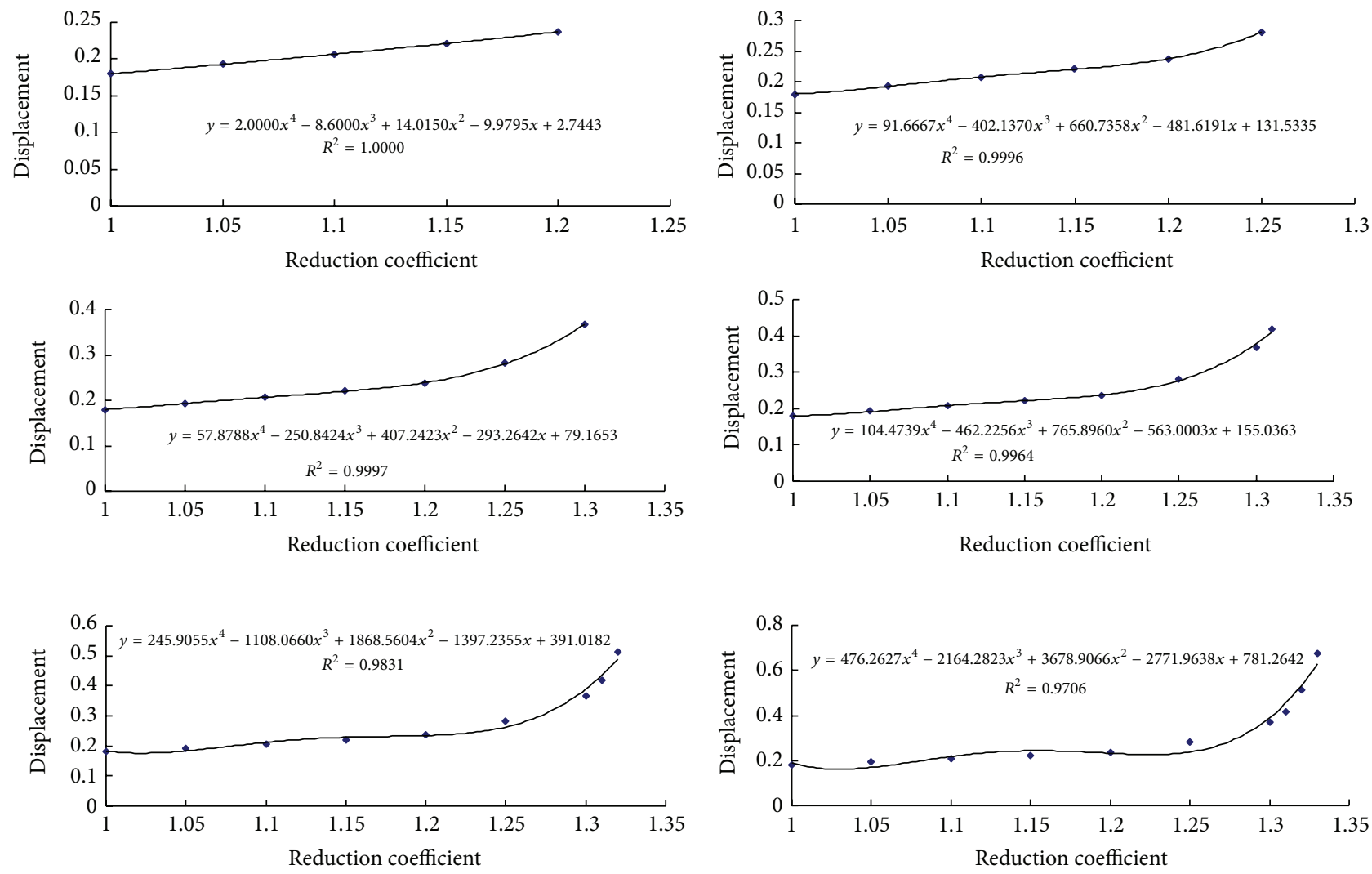

FIGURE 4: Fitting curves of displacement reduction factor under reduction factor at levels 5 to 10.

TABLE 2: Control variables $u, v$ and mutation characteristic values $\Delta$ of cusp catastrophe model under different reduction factor.

\begin{tabular}{lcccccc}
\hline$k$ & 1.3 & 1.31 & 1.32 & 1.33 & 1.34 & -0.12496 \\
\hline$u$ & 0.012125 & 0.006882 & -0.00916 & -0.04573 & -0.30447 \\
$v$ & -0.00114 & -0.00132 & -0.0025 & -0.00762 & -0.02111 & -0.05649 \\
$\Delta$ & 0.012125 & 0.006882 & -0.00916 & -0.04573 & -0.12496 & -0.30447 \\
\hline State & No mutation & No mutation & Mutation & Mutation & Mutation & Mutation \\
\hline
\end{tabular}

$x$ and establish a cusp catastrophe model on the basis of it. The reduction factor of the system should be the control factor. Thus, this paper adopts a cubic polynomial to fit the relationship between reduction factor and displacement and takes it as the equilibrium surface of the system. Obtain the system potential function by integrating the cubic polynomial, and then regularize it into the standard form of cusp model. As analyzed above, reduction factor and displacement of quartic polynomial fitting cannot be directly established. Otherwise, in the view of mathematics, the potential function of the system is the reduction factor. Under the condition of system instability, the state variable $x$ will produce a sudden jump, which will lead the potential function of the system to a sudden jump; namely the reduction factor has a sudden jump and its value is not in conformity with man-made value.

The fitting graphs are shown in Figures 5 and 7.

The potential function of the system expressed by quartic polynomial is obtained by integral of the fitting curves. Then regularize it into the standard form of cusp catastrophe model. The stability of system can be determined by catastrophe characteristic values.

When the reduction factor reaches 1.32 , the maximum slope displacement mutates (Table 2). The reason of catastrophe is due to the variation of the reduction factor (increasing gradually), resulting in the control variable of cusp catastrophe satisfying the condition of catastrophe. Even the whole system would mutate; that is, a sudden jump of the state variable (i.e., the maximum displacement of the slope) occurs. The slope jumps from an equilibrium position before instability to another equilibrium position after instability.

The cusp catastrophe model established in this paper reflects that the slope instability results from the displacement jump, which leads the status of the slope to change and the potential energy of the system also to change. (The specific physical meaning of potential energy is not clear, but the potential function is not the focus of catastrophe research.) Compared with the traditional method using displacement as potential function, cusp catastrophe model has a more 

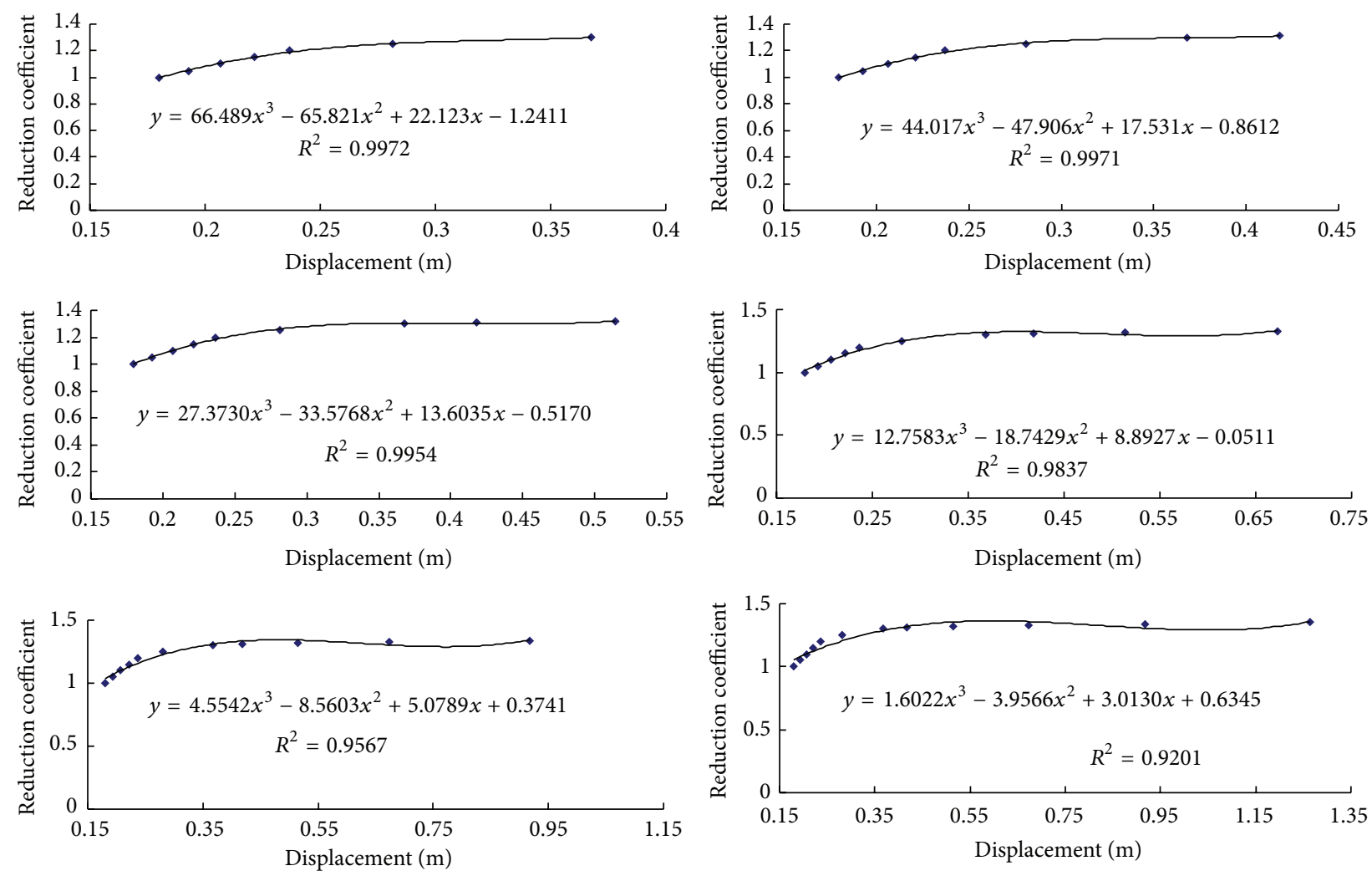

FIGURE 5: Fitting curves of displacement reduction factor under reduction factor at levels 7 to 12.

TABLE 3: Table of control variables $u, v$ and catastrophe characteristic value $\Delta$ of cusp catastrophe model under different reduction factors.

\begin{tabular}{lccc}
\hline$k$ & 1.15 & 1.2 & 1.25 \\
\hline$u$ & -0.30447 & -2.60349 & -0.03727 \\
$v$ & -0.05649 & -1.77655 & 0.003859 \\
$\Delta$ & -0.13965 & -55.959 & $-1.2 E-05$ \\
\hline State & Mutation & Mutation & Mutation \\
\hline
\end{tabular}

definite physical meaning and is consistent with practical engineering, which is established by considering reduction factor as state variable.

\section{Discussion}

4.1. The Occurrence Time of Sudden Change. When the reduction factor reaches 1.3 (reduction factor at level 7), material strength reduction factors continued being increased, and the catastrophe results of fitting curves on the basis of more than 7 groups of data $(k, u)$ can be gotten as presented in Table 1. Reduction factors and cubic fitting curves of displacement under reduction factors at levels 4 to 6 are also given in Figure 6. Likewise, transpose it to integral to get a potential function of the system fitted with quartic polynomial fit and regularize it to get the standard type of cusp catastrophe model. Thus, the stability can be determined by the catastrophe characteristic values and results are shown in Tables 3 and 4.

As is shown in Table 3, it seems that when the reduction factor reaches 1.15 (reduction factor at level 4), slope has become unstable already. However, after a careful look at fitting curves, it can be found that, at level 4, there are only four groups of data and data points are too few to use in cubic polynomial fitting. Although it can reach very high accuracy at these data points, the extension performance of fitting polynomial is very poor and it cannot reflect the actual relations between the data. When high order polynomial is adopted, more fitting points are needed to achieve a higher accuracy. It is meaningless with a few fitting points in adopting high order polynomial fitting.

4.2. Adoption of Cubic Polynomial Fitting of Conventional Catastrophe Model. The actual physical meaning of state variables and the sudden jump occurring at the time of catastrophe which results in another state of the system are considered in the catastrophe model in this paper. However, according to the analysis of calculation above, it seems that there is no way to explain the problem why calculation results are still highly accurate when adopting quartic polynomial fitting, using displacement as potential function by conventional catastrophe model and establishing the reduction 
TABLE 4: Table of control variables $u, v$ and catastrophe characteristic value $\Delta$ of cusp catastrophe model under different reduction factors.

\begin{tabular}{lcccccc}
\hline$k$ & 1.3 & 1.31 & 1.32 & 1.33 & 1.34 & 1.35 \\
\hline$u$ & 0.019105 & 0.009073 & -0.00442 & -0.01599 & -0.02413 & -0.02942 \\
$v$ & -0.04207 & -0.04198 & -0.03814 & -0.03388 & -0.03154 & -0.03091 \\
$\Delta$ & 0.047849 & 0.047586 & 0.039277 & 0.030953 & 0.026742 & 0.025597 \\
\hline State & No mutation & No mutation & No mutation & No mutation & No mutation & No mutation \\
\hline
\end{tabular}
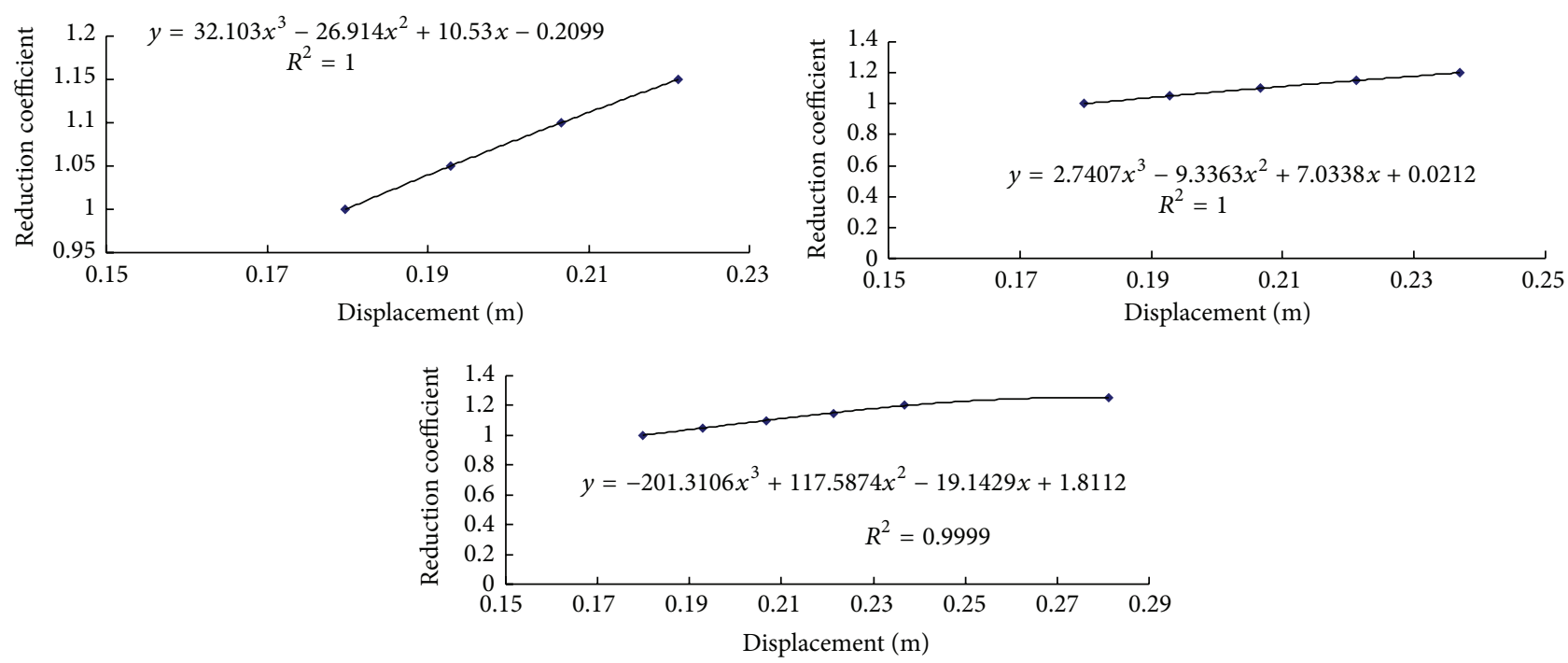

FIGURE 6: Fitting curves of the reduction factor of displacement under reduction factors at levels 4 to 6.
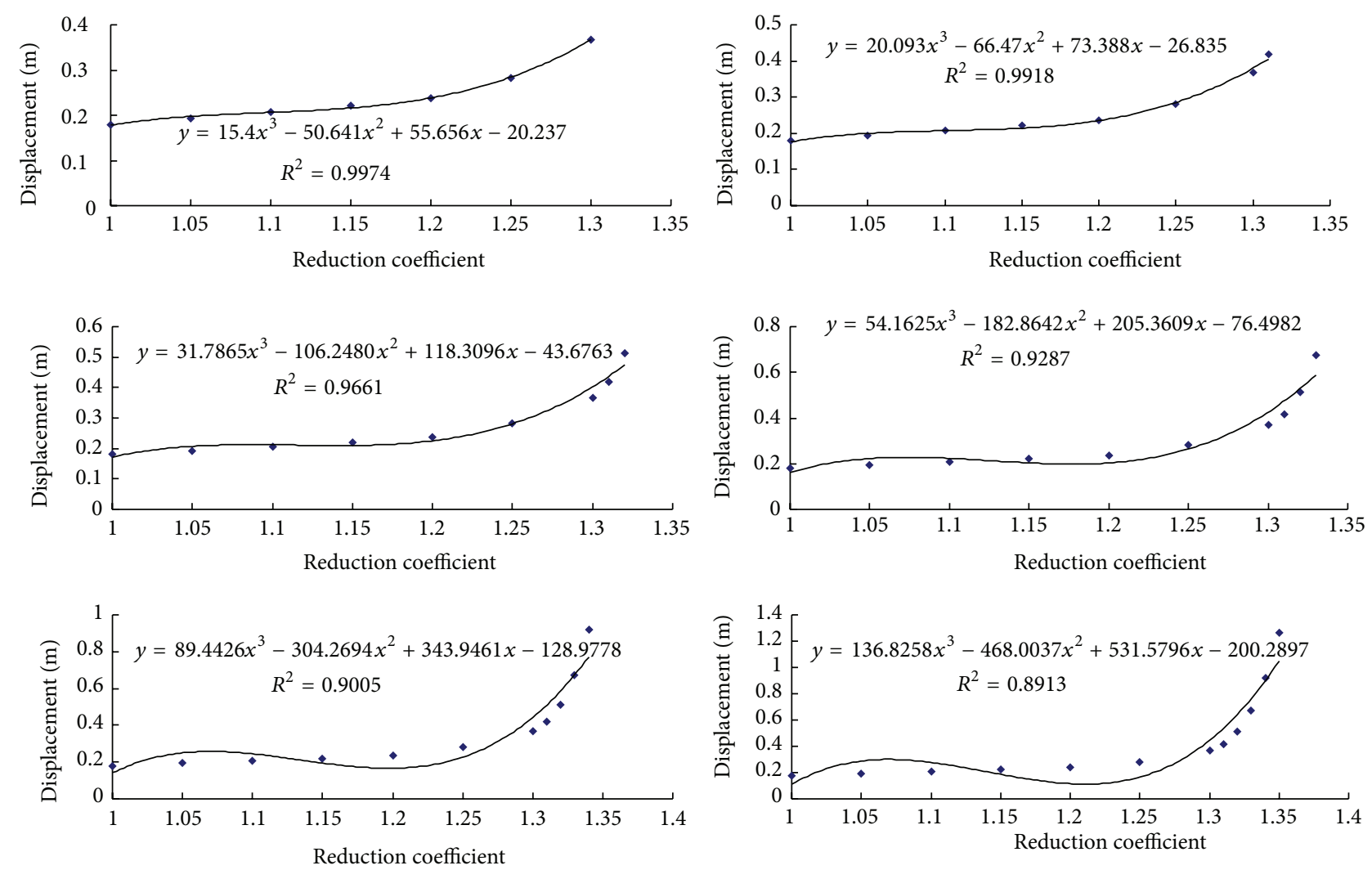

FIGURE 7: Fitting curves of the reduction factor of displacement under reduction factors at levels 7 to 12. 
factors as state variables in the catastrophe model. It is not a particular example for many studies have mentioned it before.

To make a further analysis, not only a conventional method is adopted but also a cubic polynomial fitting is used in this paper. Transpose it into integral to get reduction factors as state variables and cusp catastrophe model including displacement influence. Then determine the stability of the slope based on it.

There is an interesting phenomenon here; that is, at this point catastrophe will not happen again and slope will not lose balance (under the condition of maximum reduction factor at 1.35 which is given in this paper). It cannot give a definite conclusion only by a few examples, "reduction factors cannot be used as state variables to get the critical condition of catastrophe," but, at least, the conclusion that "when using the reduction factor as state variables a sudden jump of the reduction factor will lead to instability of slope that is not reasonable in its physical meaning" can be pointed out explicitly.

\section{Conclusion}

From the perspective of catastrophe mathematical model, a cubic polynomial data fitting is put forward to get a function as the equilibrium surface of the system in this paper. Under an excavation or loading step, the polynomial satisfies the equilibrium conditions approximately (the error is merely the fitting function error at that point). Therefore, this state is on (or near) the equilibrium surface of the potential function. The equilibrium surface obtains a system potential function expressed by a quartic polynomial through integral. After regularization, stability can be determined by the catastrophe characteristic values. This method overcomes the disadvantages of currently commonly used catastrophe criterion types which is not clear in physical meaning.

\section{Conflict of Interests}

The authors declare that they have no conflict of interests in this paper. And this research is referenced mutation in mathematics theory to solve the stability problem of geotechnical engineering. It is consistent with the main theme.

\section{Acknowledgments}

Thanks are due to National Science and Technology Support Program (2012BAK03B04/2012BAB03B02) and the Youth Foundation of National Natural Science Fund (51209078).

\section{References}

[1] G. Shao, J. Zhuo, and Q. Zhang, "Research on analysis method and criterion of rockmass stability," Chinese Journal of Rock Mechanics and Engineering, vol. 22, no. 5, pp. 691-696, 2003.

[2] X. Shan, "The possibility of tunnel rock burst occurrence by using catastrophe theory," Mine Surveying, no. 4, pp. 36-37, 2000 .
[3] C. Yan and G. Xu, "Instability analysis of deep-buried tunnels with catastrophe theory," Journal of Engineering Geology, vol. 14, no. 4, pp. 508-512, 2006.

[4] L. Wang and X. Miao, "Study of mechanism of destabilization of the mine pillar based on a cusp catastrophic model," Journal of Mining \& Safety Engineering, vol. 23, no. 2, pp. 137-140, 2006.

[5] C. Xu, Q. Ren, and R. Li, "Entropy catastrophe criterion of surrounding rock stability," Chinese Journal of Rock Mechanics and Engineering, vol. 23, no. 12, pp. 1992-1995, 2004.

[6] C.-H. Xu and Q.-W. Ren, "Criterion of entropy catastrophe of stability of surrounding rock," Rock and Soil Mechanics, vol. 25, no. 3, pp. 437-440, 2004.

[7] Y. Pan, Z.-Q. Wang, and Y. Zhang, Application of Catastrophe Theory to the Dynamic Stability Loss of Rock Mass System, Science Press, Beijing, China, 2008.

[8] Y.-Q. Lou, C.-S. Gu, and J. Li, "Study on the slope failure criterion in strength reduction finite element method based on catastrophe theory," Journal of Xian University of Architecture and Technology, vol. 40, no. 3, pp. 361-367, 2008. 


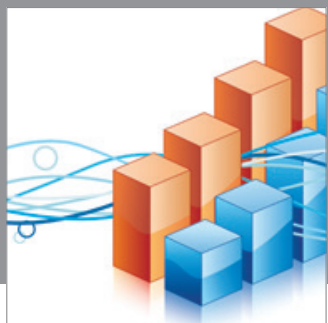

Advances in

Operations Research

mansans

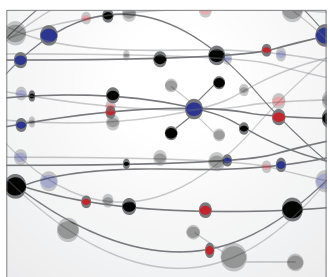

The Scientific World Journal
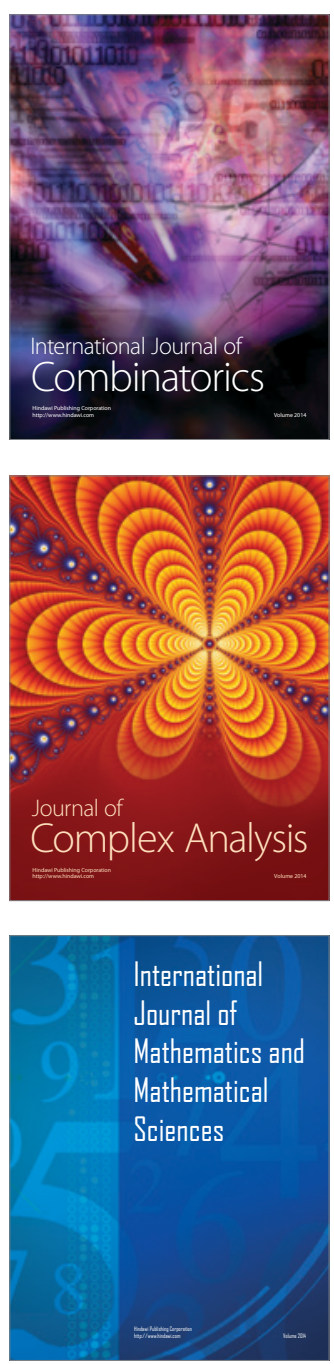
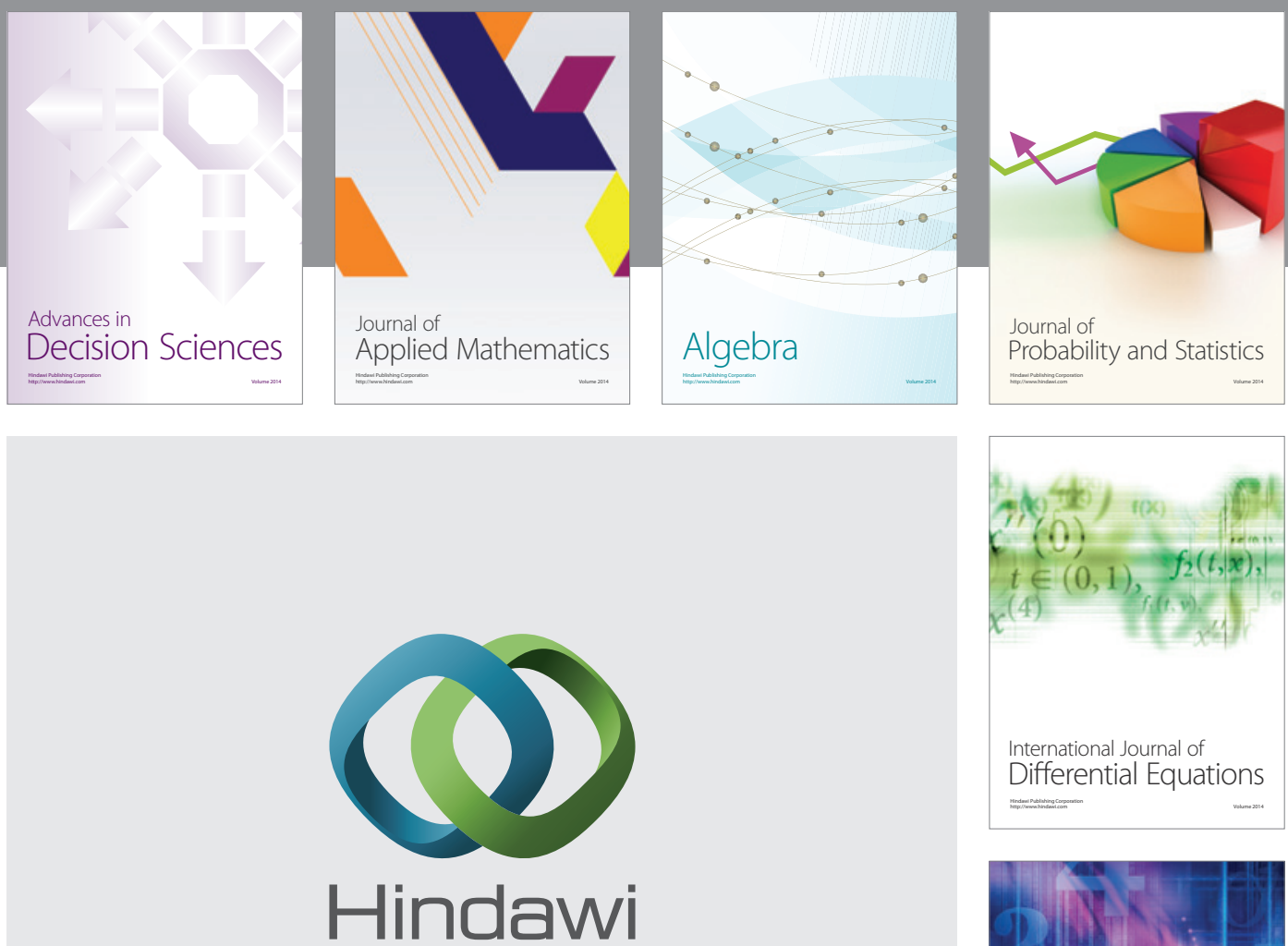

Submit your manuscripts at http://www.hindawi.com
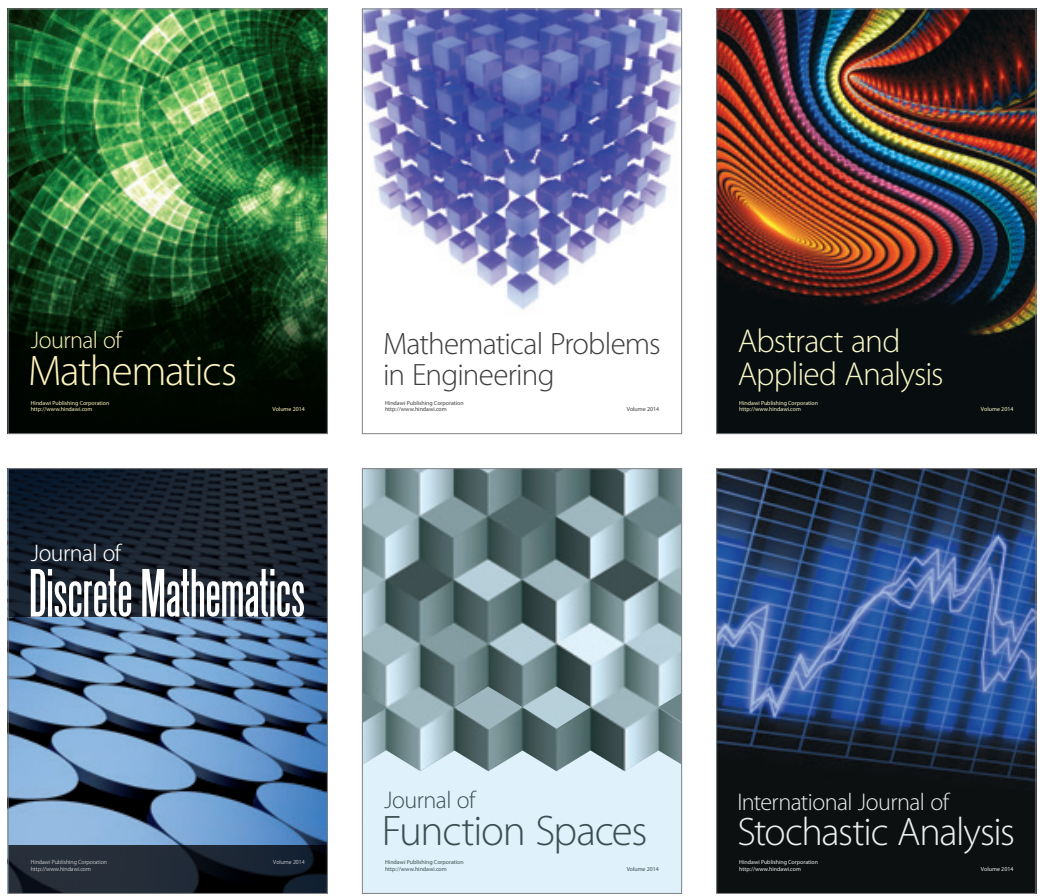

Journal of

Function Spaces

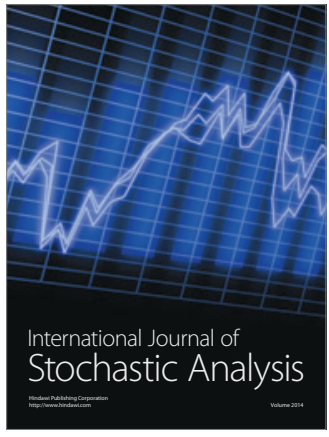

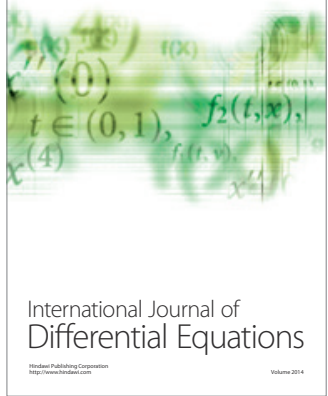
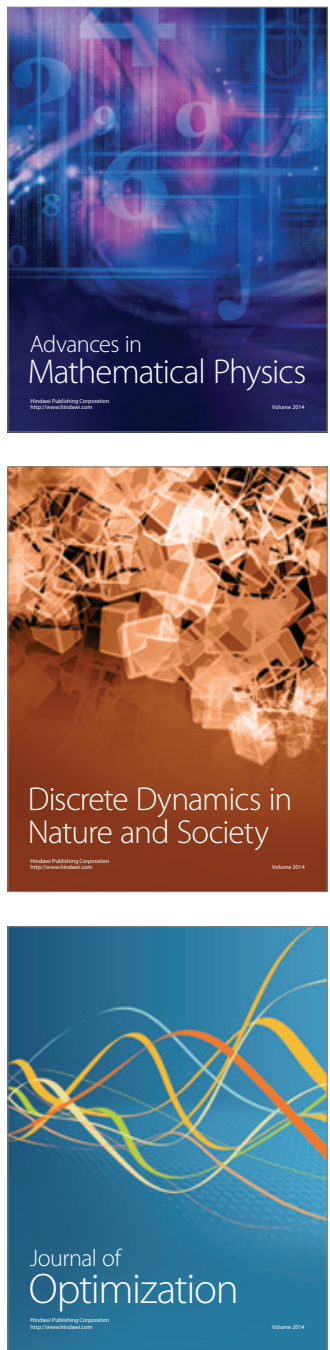